\title{
Psychiatric Genetics and Genomics
}

Peter McGuffin, Michael J. Owen, Irving I. Gottesman (Eds.)

(2002) Oxford: Oxford University Press, pp. 472, \$65 ISBN: 0-19-263 I 48-9

Psychiatric Genetics and Genomics has four parts: Basic Principles, The Genetics of Normal and Abnormal Development, The Genetics of Abnormal Behaviour in Adult Life, and Applications and Implications.

\section{Part One: Basic Principles}

The first chapter, Basic Molecular Genetics provides a very useful introduction to concepts such as recombination and the storage, transmission, and translation of information in DNA, and describes laboratory techniques such as PCR. Quantitative Genetics reviews family, twin, and adoption study methodologies, and patterns of genetic transmission, including concepts of Hardy-Weinberg Equilibrium and heritability. Linkage and Association underlines their complementarity and their differences. It includes useful sections on linkage analysis of quantitative traits, power analyses, and allelic association.

\section{Part Two: The Genetics of Normal and Abnormal Development}

Personality and Cognitive Abilities discusses different approaches to phenotype definition (dimensional, categorical), the application of DeFriesFulker extreme analysis, and the importance of animal models in investigating personality. Genetics of Mental Retardation reviews the single gene MR disorders associated with chromosomal abnormalities; the authors make the point that, although these disorders are rare, understanding their genetic basis will have relevance for the majority of (idiopathic) MR. Reading and Language Disorders reviews recent results for specific language impairment include the promising chromosome 7 linkage finding which has led to the identification of a genetic variant. Childhood Disorders includes autism (promising $7 \mathrm{q}$ finding); childhood schizophrenia (overlap with autism, and association with the chromosome 22q11deletion region); ADHD (promising association findings with DRD4 and DAT1, and the need to address issues of phenotypic definition and comorbidity); and depression (phenotypic definition and heritability of sub-groups).

\section{Part 3: The Genetics of Abnormal Behaviour} in Adult Life

Personality Disorders focuses on animal models and candidate gene studies. Using components or dimensions rather than (diagnostic) categories, knock-out mouse studies and candidate gene studies have provided converging evidence for reduced serotonergic transmission being associated with aggressive or impulsive behaviour. Genetics of Affective Disorders sets out general diagnostic and methodological challenges facing the field (including the central issue of the unknown biological validity of our psychiatric diagnostic systems); in their critical review of quantitative and molecular findings, the authors stress the need to seek genetically relevant phenotypic sub-types and to collect large, well-characterized samples to facilitate progress. Schizophrenia reviews the genetic epidemiology, phenotypic definition, and current linkage, association, and cytogenetic studies; the need for refining the phenotype is also emphasised. Substance Misuse covers alcohol dependence with linkage studies and candidate gene studies (including $\mathrm{ADH}, \mathrm{ALDH} 2$, and DRD2). Family, twin and adoption studies also establish a substantial genetic contribution to other drugs of abuse including the opiates, cannabis and cocaine. Anxiety and Eating Disorders reviews family and twin studies, and linkage, association, and animal studies, including the important rodent emotionality model of anxiety. Again, phenotypic definition and comorbidity are crucial issues, these disorders often overlapping with each other and with depression. The Dementias documents the substantial success in the application of molecular genetics: amyloid precursor protein and presenilin genes in (early onset) familial Alzheimer's; apolipoprotein $\mathrm{E}$ (and linkage findings) in late onset Alzheimer's; advances in vascular and frontotemporal dementias, Huntington's disease, and the transmissible spongiform encephalopathies are also presented.

\section{Part 4: Applications and Implications}

Pharmacogenetics studies genetic variants affecting neurotransmitter receptor systems and their predictive value for clinical treatment. With the development of high throughput technologies and large-scale SNP maps, the authors argue for a "pharmacogenomics" future with the goal of individualized treatment response. Genetic Counseling cautions that even with the substantial advances expected, it is unlikely that molecular genetic testing will be available for most psychiatric disorders, and that a more tangible benefit of available testing may be to help optimize treatment options. Ethical Considerations in Psychiatric Genetics stresses the need for researchers to inform public opinion about the outcomes and implications of genetics research. In the context of national and international ethical codes, current ethical questions of informed consent, the role of IRBs, the role of individuals and families as research participants, privacy and disclosure of test results to third parties, and commercialization of research products are discussed. The Future and Post-genomic Psychiatry sets out current progress and sketches future challenges of refining the phenotype (symptom dimensions, extreme phenotypes, endophenotypes, animal models), and identifying modifying 
genes (e.g., role of APOE4 in age of onset for Alzheimer's). Once genes are identified, implications include investigating functional variants within these genes and developing a genetic nosology.

This book provides a very comprehensive, up-to-date and timely assessment of psychiatric genetics. It critically reviews all disorders, states the progress achieved, and addresses the methodological challenges currently before the field. It also outlines prospects for future advances in a post-genomic psychiatry.
Bryan Mowry

Queensland Centre for Schizophrenia Research, The Park - Centre for Mental Health, Wacol, Australia

Email:bryan_mowry@qcsr.uq.edu.au 